\title{
Análisis y evaluación de los modelos estadísticos para el diseño de circuitos integrados
}

\section{Analysis and Evaluation of Statistical Models for Integrated Circuits Design}

\author{
Sáenz-Noval J.J. \\ Grupo de diseño de circuitos integrados CIDIC \\ Escuela de Ingeniería Eléctrica y Electrónica \\ Universidad Industrial de Santander, Bucaramanga, Colombia \\ E-mail: jsaenz@lsi.usp.br
}

\author{
Roa-Fuentes E.F. \\ Grupo de diseño de circuitos integrados CIDIC \\ Escuela de Ingeniería Eléctrica y Electrónica \\ Universidad Industrial de Santander, Bucaramanga, Colombia \\ E-mail:elkim@purdue.edu
}

Información del artículo: recibido: agosto de 2009, reevaluado: febrero y mayo de 2010, aceptado: noviembre de 2010

\section{Resumen}

Los modelos estadísticos para circuitos integrados (CI) permiten estimar antes de la fabricación el porcentaje de dispositivos aceptables en el lote de fabricación. Actualmente, Pelgrom es el modelo estadístico más aceptado en la industria; sin embargo, se derivó de una tecnología micrométrica, la cual no garantiza confiabilidad en los procesos de fabricación nanométricos. Este trabajo considera tres de los modelos estadísticos más relevantes en la industria y evalúa sus limitaciones y ventajas en el diseño analógico, de manera que el diseñador tenga un mejor criterio en su elección. Además, se muestra cómo pueden utilizarse varios modelos estadísticos para cada una de las fases y propósitos de diseño.

\section{Abstract}

Statistical models for integrated circuits (IC) allow us to estimate the percentage of acceptable devices in the batch before fabrication. Actually, Pelgrom is the statistical model most accepted in the industry; however it was derived from a micrometer technology, which does not guarantee reliability in nanometric manufacturing processes. This work considers three of the most relevant statistical models in the industry and evaluates their limitations and advantages in analog design, so that the designer has a better criterion to make a choice. Moreover, it shows how several statistical models can be used for each one of the stages and design purposes.

\section{Descriptores}

- mismatch

- diseño analógico

- variabilidad

- rendimiento

- modelos

- reducción del canal

\section{Keywords}

- mismatch

- analog design

- variability

- yield

- models

- channel shrinkage 


\section{Introducción}

El mismatch en los parámetros de un transistor MOS se considera uno de los factores limitantes en el desempeño de los circuitos analógicos. Conforme la tecnología avan$\mathrm{za}$, las dimensiones de los transistores alcanzan escalas atómicas, produciendo una baja repetibilidad y una alta incertidumbre en su comportamiento eléctrico. Pero el problema principal no radica en la alta variabilidad ligada a los nuevos procesos tecnológicos, sino en la incertidumbre para predecirlos y modelarlos adecuadamente. El modelo del descasamiento simplista, el cual es inversamente proporcional al área, ha demostrado deficiencias para escalas de integración sub-micrón; además éste no es consistente con todos los parámetros físicos del proceso, debido a que cada uno de estos exhibe una dependencia diferente con el dimensionamiento y la polarización. Esto lo hace un modelo altamente inadecuado para la validación final de un CI.

Se ha propuesto un conjunto de nuevos modelos de mismatch, pero muchos presentan deficiencias en su implementación como son tiempo de cómputo y complejidad de sus expresiones (Croon, 2002) y (Drennan, 2003). Frecuentemente, la aplicabilidad de modelos estadísticos de alta correlación con los resultados de silicio, representa un compromiso en tiempo de cómputo, además los hacen prácticamente inutilizables para los cálculos manuales en el diseño. Al no tener un estimativo correcto del mismatch, el diseñador se ve obligado a determinar a ciegas el rendimiento del circuito. La desestimación de las geometrías del transistor produce amplias desviaciones en sus parámetros eléctricos. Por otro lado, el uso de geometrías de transistor conservadoras tiene como consecuencia el desaprovechamiento del área que además conlleva al aumento de las capacitancias (Serrano-Gotarredona, 2004). Este tipo de compromisos entre el desempeño y el rendimiento de los circuitos debe manejarse a través de modelos estadísticos de alta exactitud en etapas previas a la fabricación.

Una correcta selección de modelos, tanto determinísticos como estadísticos, dependen de la etapa y los propósitos de diseño sobre los que se trabaje. Por ejemplo, en etapas iniciales del diseño es común utilizar modelos con descripciones comportamentales del sistema, pues es primordial analizar su funcionamiento antes que su robustez. Sin embargo, el objetivo del diseño muda conforme la topología y las variables de diseño se establecen y el análisis de robustez va tornándose importante, llegando a ser el más relevante durante el cierre del proceso de diseño de un circuito integrado. Este trabajo pretende evaluar las fortalezas y deficiencias de cada uno de los modelos considerados más relevantes en la actualidad, estableciendo seis criterios de evaluación. El propósito es guiar al diseñador en la selección de los modelos estadísticos usados en cada una de las fases de diseño y extender los análisis de variabilidad a las etapas más primarias del diseño como son: la selección de la topología y el dimensionamiento. El establecimiento de los criterios de evaluación y la fundamentación teórica de los tres modelos a usar serán expuestos en la siguiente sección. Posteriormente, se muestra un análisis detallado de cada uno de los modelos seleccionados. Por último, los aspectos y afirmaciones más relevantes se condensan como conclusiones en la última parte del trabajo.

\section{Modelos de mismatch}

A la hora del análisis del fenómeno del mismatch en los circuitos integrados, este trabajo plantea seis criterios de evaluación que se deben tener en cuenta. Estos criterios son:

\section{Significado físico}

El mismatch se origina por variaciones en los parámetros físicos inherentes al transistor. Cuando se hace la formulación del mismatch en función de parámetros eléctricos correlacionados entre sí o factores de ajuste de modelado, origina una sobre-estimación en la variabilidad del dispositivo; es así como un modelo acertado debe fundamentarse en la variación de los parámetros físicos esenciales.

\section{Continuidad}

La continuidad propia a los procesos físicos ligada al comportamiento del transistor, los posibilita para modelarse matemáticamente por funciones igualmente continuas. Además, dada la consigna de baja potencia y bajo voltaje en el diseño, el modelo debe incluir regiones como la sub-umbral y la inversión moderada. Para esto, el mismatch debe replantearse como un modelo eléctrico continuo en función de la polarización.

\section{Medibilidad}

Muchos de los parámetros físicos de un transistor no pueden ser directamente medibles, por lo que muchas veces se requiere un ajuste matemático en el proceso de modelado o una medición indirecta del mismo. Un modelo altamente medible debe establecer un conjunto de pruebas que garanticen resultados repetibles y una medición precisa de los parámetros físicos que involucra. 


\section{Precisión}

Capacidad de predecir con bajas tasas de error el comportamiento tanto determinístico como aleatorio de cada uno de los parámetros eléctricos. El comportamiento determinístico será relevante en la estimación del desempeño del circuito, en contraste con el comportamiento estocástico, el cual será útil para la estimación del rendimiento.

\section{Utilidad}

Un modelo estadístico preciso no garantiza su fácil aplicabilidad en el flujo de diseño, ni mucho menos una utilización en cálculos manuales. Los modelos que precisen pocos parámetros, expresiones matemáticas simples y tiempos de convergencia bajos son los más aptos para el diseño. Además, deben establecer los componentes que serán incluidos en simulaciones pre-layout ${ }^{1}$ y en la extracción pos-layout.

\section{Adaptabilidad}

Se debe garantizar que el modelo sea independiente del tipo de proceso utilizado y de la longitud mínima de canal. En consecuencia, debe permitir la inclusión de nuevos fenómenos físicos sin un drástico cambio en su formulación.

El tema del mismatch ha empezado a ser recurrente en el diseño analógico, llegándose a publicar más de una docena de modelos al respecto. El modelo que se considera pionero fue propuesto en Lakshmikumar (1986) y brindó un punto de partida para el tratamiento y derivación matemática del fenómeno aplicado al diseño. Basados en este trabajo surgieron modelos como el de Pelgrom (1989), el cual sería ampliamente aceptado por la industria. Trabajos posteriores se pueden considerar ampliaciones de éste, en donde se incluían los efectos de la reducción del canal que comenzaban a ser relevantes en procesos de escala nanométrica. Luego surgen trabajos como los de Serrano (2004) y Galup (2005), que tienen la ventaja de modelar continuamente el mismatch del transistor MOS. Hasta el momento el único modelo que se ha mantenido como estándar en la industria es Pelgrom, pero las exigencias actuales de confiabilidad en los dispositivos requieren un inminente cambio.

El estudio de los modelos conduce a tres trabajos que se consideran fundamentales, relevantes e innovadores

\footnotetext{
${ }^{1}$ Término en inglés utilizado para referir la representación gráfica de la disposición de las máscaras empleadas en el proceso de fabricación de un CI.
}

en el análisis y determinación del mismatch. El objetivo es exponer las ideas fundamentales de cada uno de estos tres modelos, para luego establecer en la sección de Discusión un análisis bajo los seis criterios anteriores.

\section{Modelo de Pelgrom (1989)}

Propuesto en (Pelgrom, 1989), se convirtió en el primer modelo de mismatch estandarizado por la industria, debido a su precisión y aplicabilidad adecuadas a las escalas de integración de su momento. Por primera vez se distinguen matemáticamente las variaciones de proceso globales y locales. Además, se reconocen algunas de las causas físicas que originan el mismatch en la corriente. Sobre estas consideraciones, se establece una discusión basada en el análisis y las mediciones del voltaje umbral, el factor de corriente y el factor de substrato del transistor MOS como función del área, la distancia y la orientación del transistor. Su correcto análisis, medición y extrapolación lo convirtieron en el modelo más representativo del mismatch y uno de los trabajos más indexados en el área de la microelectrónica.

Para Pelgrom, el valor de un parámetro $P$ está compuesto por un término constante y otro variable aleatoriamente. Para el caso estocástico, se establecen variaciones locales como ruido blanco espacial, caracterizado por una baja correlación con la distancia. Conjuntamente, dado que la ubicación del $\mathrm{die}^{2}$ en la oblea antes del empaquetado es desconocida, el efecto de la distribución circular en el mismatch puede ser modelado como un proceso estocástico adicional de una gran correlación con la distancia entre dos transistores. De esta manera, Pelgrom define la varianza en la desviación del parámetro $P$ de dos transistores con ancho $W$, longitud $L$ y espaciamiento $D_{x}$ a lo largo de $x$, como:

$\sigma^{2}(\Delta P)=\frac{A_{P}^{2}}{W L}+S_{P}^{2} D_{x}^{2}$

donde $A_{p}^{2}$ es la constante de proporcionalidad con el área para el parámetro $P$, mientras que $S_{P}$ describe la variación del parámetro $P$ con el espaciamiento $D_{x}$. Las constantes de proporcionalidad se definen por medio de la varianza de los parámetros. Cualitativamente, las variaciones locales disminuyen a medida que el tamaño del dispositivo aumenta, puesto que los parámetros se promedian sobre una mayor área o distancia.

\footnotetext{
${ }^{2}$ Un die es un complejo conjunto de componentes electrónicos y sus interacciones impresos y enclavados en una pequeña pieza de material semiconductor.
} 
Pelgrom establece la expresión del mismatch en la corriente de drenador entre dos transistores como

$\frac{\sigma_{I_{D}}^{2}}{I_{D}^{2}}=\frac{4 \sigma_{V_{t}}^{2}}{\left(V_{G S}-V_{t}\right)^{2}}+\frac{\sigma_{\beta}^{2}}{\beta^{2}}$

\section{Modelo de Drennan (2003)}

Usado exclusivamente durante varios años por Motorola, este modelo surge como respuesta a una serie de defectos en la implementación y estimación del mismatch por Pelgrom. Intenta describir en un sentido más físico este fenómeno, obteniéndose así un modelo aplicable a un amplio rango en la polarización y las condiciones de geometría del transistor. Discrepa en que el aumento del área en todos los casos genera una inminente disminución del mismatch.

Para el modelado, Drennan considera dos tipos de parámetros: parámetros de proceso y parámetros eléctricos. Los parámetros de proceso son aquellos físicamente independientes de los parámetros que controlan el comportamiento eléctrico del dispositivo. Por su parte, los parámetros eléctricos son los parámetros de interés para el diseñador.
La tabla 1 contiene ejemplos de parámetros de proceso incluidos en este modelo y los parámetros eléctricos relevantes. Por ejemplo, el voltaje umbral $V_{t}$ no es un parámetro de proceso, ya que depende de $V_{f b}, t_{o x}$, $N_{\text {sив }}, L$ y $W$. El parámetro $V_{t}$ exhibe una dependencia directa con $V_{f b}$ y $t_{o x^{\prime}}$ inversa con $W$ y $L$ y logarítmica con $N_{\text {SUB. }}$. Lo anterior significa que la estimación de la varianza de vía la relación Pelgrom es física y matemáticamente incorrecta.

Fundamentado en la dependencia de la varianza de cada parámetro con el dimensionamiento, en conjunto con la teoría de propagación de la varianza (POV) (Lei, 1998), Drennan establece la ecuación (3).

El signo $(\sim)$ en la tabla indica una variable normalizada. El vector del lado izquierdo de (3) es un conjunto de $n$ desviaciones estándar recolectadas de muchos dies, en varias condiciones de polarización y geometría. La combinación de las condiciones se establece de manera que cada una de las varianzas sea medida en sus casos más significativos. La matriz en (3), contiene el cuadrado de las sensibilidades de $I_{d}$, con respecto a cada uno de los parámetros de proceso. Estas sensibilidades son numéricamente evaluadas usando SPICE en las condiciones de geometría y polarización correspondientes a la medida.

\begin{tabular}{ll}
\hline \multicolumn{1}{c}{ Parámetros de proceso } & \multicolumn{1}{c}{ Parámetros eléctricos } \\
\hline Voltaje de banda plana $\left(V_{f b}\right)$ & Corriente de drenador $\left(I_{D}\right)$ \\
Movilidad de portadores $(\mu)$ & Voltage puerta-fuente $\left(V_{G S}\right)$ \\
Concentración de dopantes del substrato $\left(N_{S U B}\right)$ & Transconductancia principal $\left(g_{m}\right)$ \\
Desviación de longitud de canal $(\Delta L)$ & Transconductancia de salida $\left(g_{0}\right)$ \\
Desviación del ancho $(\Delta W)$ & \\
Efecto de canal corto en $\mathrm{Vt}\left(V_{t t}\right)$ & \\
Efecto de la estrechez del ancho en $\mathrm{Vt}\left(V_{t w}\right)$ & \\
Espesor del óxido de puerta $\left(t_{o x}\right)$ & \\
Resistencia de drenador-fuente $\left(\rho_{s h}\right)$ & \\
\hline
\end{tabular}

Tabla 1. Parámetros eléctricos y de proceso más relevantes en el mismatch

$$
\begin{aligned}
& {\left[\begin{array}{c}
\sigma_{\tilde{I}_{d 1}}^{2} \\
\sigma_{\tilde{I}_{d 2}}^{2} \\
\sigma_{\tilde{I}_{d 3}^{2}}^{2} \\
\vdots \\
\sigma_{\tilde{I}_{d n}}^{2}
\end{array}\right]=}
\end{aligned}
$$

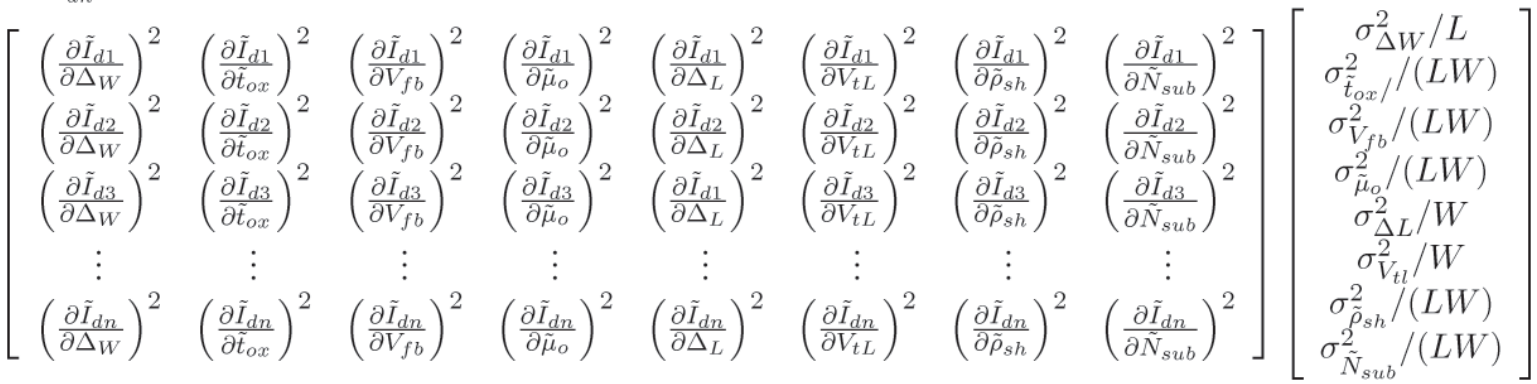


Determinados los valores de las dos matrices en (3), el vector del lado derecho puede calcularse usando una regresión lineal. Básicamente, cada parámetro se supone independiente, pero si el método determina una correlación significativa entre éstos, implicará que ha sido tomado un conjunto incorrecto de parámetros.

\section{Modelo Galup-Montoro (2007)}

Este modelo fue propuesto como parte integral de una nueva generación de modelos compactos, que tienen la ventaja de modelar continuamente la operación del transistor desde inversión débil hasta inversión fuerte. La derivación de sus expresiones se hace a partir del modelo eléctrico planteado en (Galup, 2007) denominado ACM (Advanced Compact Model). Dado a que el impacto de las fluctuaciones locales de dopantes en el mismatch son significativas (Yang, 2003), este modelo deriva sus expresiones basado en la teoría de la fluctuación en el número de portadores, usada también para determinar el ruido $1 / f$ del transistor en el modelo $A C M$.

Las mediciones hechas por trabajos anteriores (Croon, 2002) y (Serrano, 2004) han mostrado una alta dependencia de la corriente con las variaciones en $V_{t}$, las cuales se atribuyen ampliamente a las fluctuaciones en los dopantes. Las fluctuaciones en la corriente de drenador resultan de la suma de todas las fluctuaciones locales a lo largo del canal. Dada la no-uniformidad en los efectos del canal, el transistor se modela como tres dispositivos dispuestos en serie como se muestra en la figura 1a: un transistor superior, un transistor inferior y un pequeño elemento de canal de longitud $\Delta x$, representado por una resistencia. El propósito de esta fragmentación consiste en promediar de una manera más acertada los efectos individuales subyacentes al canal.

Existen básicamente dos tipos de fluctuaciones de los dopantes: las globales y las locales. Las primeras se refieren al valor promedio a lo largo del canal, y son las responsables de establecer el denominado perfil de dopaje como se muestra en la figura $1 \mathrm{~b}$, el cual es altamente dependiente de la tecnología. Mientras tanto, las fluctuaciones de dopantes locales ocurren en pequeñas secciones del canal, sumándose en forma de ruido blanco a lo largo del perfil de dopaje (Yang, 2003).

Para modelar matemáticamente el efecto del perfil de dopaje, se define la constante como el valor que pondera verticalmente la deflexión de carga, matemáticamente sería:

$$
N_{c i}=\int_{0}^{y_{d}} N_{A}\left(1-\frac{y}{y_{d}}\right)^{2} d y
$$

donde $N_{A}$ es la concentración neta de dopantes tanto aceptores como donadores en un volumen elemental de la capa de deflexión.

Galup-Montoro (2007) establece que la varianza de la corriente de un transistor MOS en sub-umbral, saturación o tríodo en términos de parámetros del modelo $A C M$ está determinada por:

$$
\frac{\sigma_{I_{D}}^{2}}{I_{D}^{2}}=\frac{N_{c i}}{W L N^{* 2}} \frac{1}{i_{f}-i_{r}} \ln \left(\frac{1+i_{f}}{1+i_{r}}\right)
$$

donde $N^{*}$ se define como:

$$
N^{*}=\frac{n C_{o x} \phi_{t}}{q}
$$

La ecuación (5) puede simplificarse para unas condiciones específicas. En el caso de inversión débil, $i_{f} \cong i_{r} \mathrm{y}$ $i_{f}<1$, así (5) se convierte para inversión débil en:

$\frac{\sigma_{I_{D}}^{2}}{I^{2}{ }_{D}}=\frac{N_{c i}}{W L N^{* 2}}$

Incluyendo los efectos de primer orden en la variación de la movilidad, el grosor del óxido de puerta y el factor de pendiente se define $B_{I_{S 0}}$, incluyendo este factor en la ecuación (5), para toda región se tiene

$$
\frac{\sigma_{I_{D}}^{2}}{I^{2}{ }_{D}}=\frac{1}{W L}\left[\frac{N_{c i}}{N^{* 2}} \frac{1}{i_{f}-i_{r}} \ln \left(\frac{1+i_{f}}{1-i_{f}}\right)+B_{I_{S Q}}\right]
$$

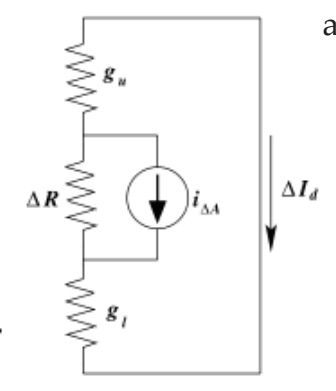

a)

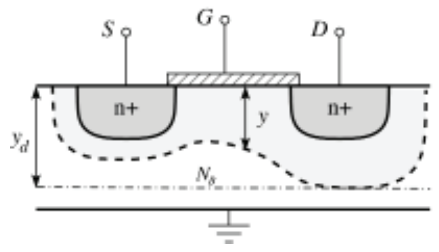

b)

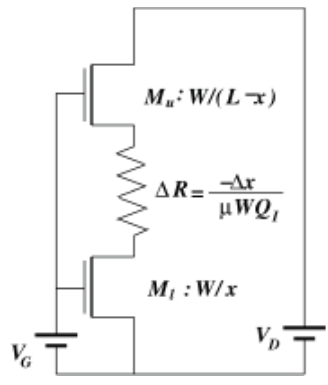




\section{Discusión}

Esta sección se dedica a discutir los tres modelos de mismatch antes mencionados bajo cada uno de los seis criterios establecidos. Se pretende así brindar un enfoque crítico al modelado estadístico y la estimación del rendimiento en circuitos analógicos, con el fin de guiar la elección del modelo a utilizar en los distintos contextos del diseño de los CI.

\section{Significado físico}

Pelgrom establece los componentes físicos mutuamente independientes que influyen en el factor de corriente $\beta$, pero tal consideración no fue tomada para la deducción de la varianza de $V_{t}$. El voltaje umbral es dependiente de $V_{f f}, W, L, t_{\text {ox }}$ y $N_{\text {SuB }}$; por su parte, el factor de corriente depende de $W, L$, y $N_{S U B}$, en consecuencia, existiría una correlación entre la varianza de $\beta$ y $V_{t}$ a través de $W, L$ y $t_{o x}$. La ecuación (2) al despreciar los términos comunes entre las dos varianzas, está sobre-estimando el mismatch de la corriente, en conjunto con su derivación del modelo cuadrático de nivel 1 , lo cual la convierte en una estimación poco confiable. Por su parte, Drennan (2003) teniendo en cuenta esta sobre-estimación, redefine (2) como:

$$
\frac{\sigma_{I_{D}}^{2}}{I_{D}^{2}}=\frac{\sigma_{\beta}^{2}+4 \sigma_{V_{t o}}^{2}}{\left(V_{G S}-V_{t}\right)^{2}}
$$

La expresión anterior presenta una inconsistencia de unidades para que sea coherente a este respecto, la varianza del factor de corriente $\sigma_{\beta}^{2}$ debería estar en $\left[V^{2}\right]$. En la figura 2 se contrastan las expresiones (2) y (9) junto con la estimación de la varianza de la corriente $\sigma_{I_{D}}$ bajo el modelo BSIM3v3. Para este propósito se variaron UO, TOX y VTHO fundamentados en la información extraída de los modelos worst-case $e^{3}$. Luego el resultado se contrastó con el cálculo de las expresiones teóricas de primer nivel. Es interesante destacar la sobre-estimación de las dos expresiones teóricas para valores bajos, debido a las diferencias con BSIM3v3, pero (9) comienza a ser un buen estimativo de la varianza de la corriente para tensiones mayores a $0,3[\mathrm{~V}]$. Por su parte, la expresión (2) de Pelgrom es un mejor tasador de la varianza de la corriente del modelo BSIM, que la ecuación de Drennan. Siguiendo el esquema de propaga-

\footnotetext{
${ }^{3}$ Entre los modelos worst-case están: típico, peor caso en velocidad, peor caso en potencia, $\mathrm{y}$ a nivel digital peor caso del cero y el uno.
}

ción de la varianza establecido por Drennan se producen los mismos resultados que (2), por lo que la expresión (9) carece de contexto.

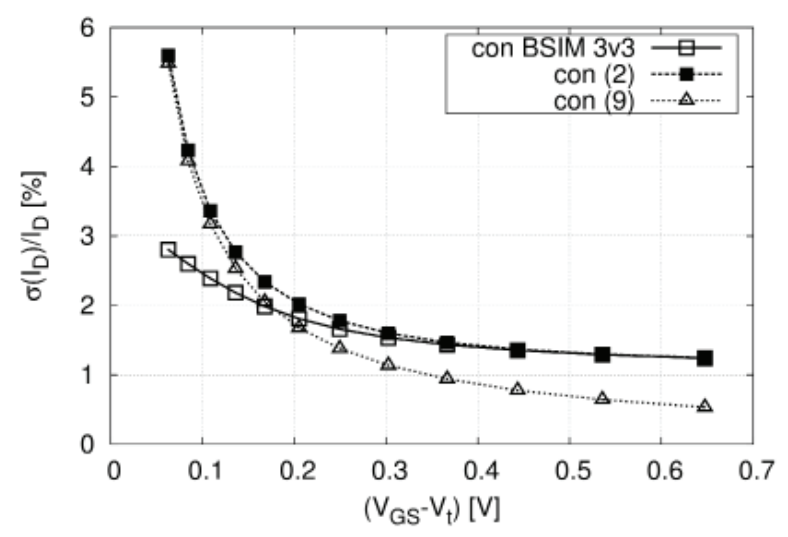

Figura 2. Mismatch de $I_{D}$ vs. $V_{G S}-V_{t}$

En el caso de Drennnan se distinguen correctamente los parámetros de proceso mutuamente independientes, pero es el modelo de simulación el que determina su correlación por medio de un análisis de sensibilidad. A pesar de que los resultados de las sensibilidades sean medianamente dependientes del error tolerable y el paso de simulación, trabajar directamente sobre el modelo comportamental trae beneficios como la reducción del error de estimación. Otro aspecto importante de esta formulación consiste en el carácter más físico que adquiere la dependencia de los parámetros a la contribución total del mismatch, gracias a la teoría de la propagación de la varianza, la cual indaga directamente la dependencia en la ecuación comportamental. Para Pelgrom, éste era un término constante dividido por el área, sin distinción alguna del parámetro tratado. Por ejemplo, el factor de corriente $\beta$ tiene una dependencia inversa con respecto al ancho $W$, pero esta característica no es evidenciada en el cálculo de $\sigma_{\beta}$, donde se observa una dependencia proporcional.

En el trabajo de Galup, las ecuaciones se fundamentan en un modelo totalmente físico y continuo: el modelo $A C M$. Las derivaciones a pesar de que no incluyen los efectos en la movilidad, el óxido de puerta, o la pendiente de sub-umbral, son coherentes y detallistas. Una amplia deducción matemática se lleva a cabo para tomar en cuenta sólo un factor de mismatch: el efecto de fluctuación de los portadores locales, lo cual mediría lo complejo que podrían resultar futuras inclusiones. Se evidencia un problema que surge de la suposición del parámetro $N_{o i}$ como una cantidad constante. Al parámetro $N_{o i}$ están ligados fenómenos despreciados en la derivación del modelo, los cuales están relacionados con el efecto cuerpo y el efecto de la tensión de drenador- 
fuente sobre el perfil de dopaje y la tensión umbral denominado DIBL (Drain Induced Barrier Lowering), entre otros. Un mejor modelado podría llevar a expresiones más complejas pero que le darían mayor significado físico al modelo.

\section{Continuidad}

La deducción del modelo Pelgrom fue hecha exclusivamente para transistores con canal invertido, por lo que sub-umbral está excluido. Debido a esto se intentará ajustar la expresión (2) de la manera más general, con el propósito de lograr incluir sub-umbral en ésta. Otra forma de expresar la varianza de la corriente despreciando los efectos de $\beta$ sería:

$\frac{\sigma_{I_{D}}^{2}}{I_{D}^{2}}=\frac{A_{V_{t}}^{2}}{W L}\left(\frac{g_{m}}{I_{D}}\right)^{2}$

En principio, (10) definiría la varianza de la corriente de un transistor MOS en un amplio rango de polarización que incluye inversión débil, por lo tanto, Pelgrom sería continuo ¿Pero (10) es una expresión adecuada para $\sigma_{I_{D}}$ ? La respuesta es sí. Utilizando el concepto de propagación de la varianza usado en (Drennan, 2003) se tiene:

$$
\frac{\sigma_{I_{D}}^{2}}{I_{D}^{2}}=\frac{1}{I_{D}^{2}} \frac{\partial I_{D}^{2}}{\partial V_{t}} \sigma_{V_{t}}^{2}
$$

Pero la derivada de la corriente $I_{D}$ con respecto al voltaje umbral $V_{t}$ tanto en sub-umbral como en canal invertido es igual a $-g_{m}$. Por lo tanto (11) se convierte en:

$$
\frac{\sigma_{I_{D}}^{2}}{I_{D}^{2}}=\left(\frac{g_{m}}{I_{D}}\right)^{2} \sigma_{V_{t}}^{2}
$$

la cual corresponde a la misma expresión en (10). De esta manera, se establece una continuidad del modelo Pelgrom, válida únicamente para un $\sigma_{I_{D}}$ dependiente tan sólo del mismatch en $V_{t}$. Cabe aclarar que la continuidad está limitada a la derivación de la varianza de la corriente por medio de la ecuación cuadrática básica. Para otras ecuaciones y parámetros, es posible que el modelo Pelgrom no sea continuo.

La expresión de Drennan es tan continua como el modelo de simulación usado para calcular las sensibilidades. Para el caso de BSIM3v3, el cual está definido por partes; inicialmente las ecuaciones garantizan una continuidad en el rango de la polarización para un dispositivo con dimensiones estáticas, que luego se verifica calculando el error relativo entre dos iteraciones, situación que se repite en el cálculo de las sensibilidades. Pero la continuidad de los parámetros de este modelo, como la pendiente de sub-umbral $n$ y el coeficiente $D I B L$ en sub-umbral, estaría ampliamente limitada, dado que éstos tan sólo aparecen o son relevantes para ciertas regiones de operación. La continuidad de este modelo no es dependiente sólo del modelo de simulación, sino del conjunto de datos recolectados, pues el cálculo de cada una de las varianzas de los parámetros debe converger a un valor constante para distintas condiciones de dimensionamiento y polarización. En conclusión, se podría afirmar que el modelo de Drennan es condicionalmente continuo.

El modelo de Galup-Montoro es continuo, pues apoya toda su deducción sobre la base de un modelo continuo como el modelo $A C M$, del cual propone una expresión para el mismatch, que es válida para un amplio intervalo de polarizaciones. Ésta es una gran ventaja en la medida en que el mismatch pasa a ser una expresión determinista como lo es la corriente. Posiblemente la inclusión de fenómenos despreciables en la deducción del modelo garanticen su continuidad.

\section{Medibilidad}

Una cualidad importante del modelo de Pelgrom es su procedimiento original de medida, ya que se calculan tanto los parámetros eléctricos $\left(V_{t}, \beta, K, \theta\right)$ como sus varianzas en un proceso donde se involucran varios lotes durante varios años. Por lo tanto, la credibilidad de los datos es alta. El cálculo de los parámetros eléctricos fue realizado por medio del ajuste matemático, y partiendo de la expresión cuadrática de la corriente, válida sólo para canales largos. En el contexto de Pelgrom, el error inherente a la aplicación de la ecuación cuadrática es bajo, dado que se trata de un proceso de $1,6 \mu \mathrm{m}$, pero para escalas menores se hace imprescindible el uso de las ecuaciones comportamentales, que incluyen un conjunto más amplio de parámetros. De esta manera, los procesos de ajuste de parámetros se van haciendo más tediosos, por lo cual comúnmente se ha optado por el análisis y la medición de un único parámetro eléctrico como la corriente. En consecuencia, el valor de las constantes del modelo Pelgrom para cada parámetro es fácilmente calculable dada la simplicidad de la ecuación (1). La adopción de la corriente como parámetro esencial en la medición del mismatch es obvia, ya que el transistor MOS es un transconductor.

El proceso de medida en el modelo Drennan parte de una correcta inclusión de parámetros de proceso altamente dependientes, de manera que estos no se vean correlacionados entre sí. La etapa más crítica consiste 
en la selección de las dimensiones y condiciones de polarización que resultan relevantes para cada parámetro, y que permitirían calcular la varianza relacionada a ese parámetro. Esto representa un inconveniente alto para aquellos conjuntos de valores donde dos o más parámetros son significativos al mismatch total, pues se requerirían al menos un número de muestras iguales al número de parámetros relevantes en una determinada condición de polarización. El diseñador está lejano de percibir estos casos excepcionales, por lo que el cálculo de la regresión matemática determinaría erróneamente las varianzas, en el mejor caso donde no se encuentre una singularidad en la matriz (3). Por lo tanto, se requiere un alto grado de conocimiento del proceso para efectuar una medición correcta del modelo de Drennan.

La caracterización de una tecnología bajo el modelo de mismatch de Galup-Montoro implica la estimación de dos valores característicos: $N_{c i}$ y $B_{I_{s 0}}$. Por lo tanto, el proceso de caracterización basado en una regresión matemática es sencillo y preciso. El modelo de Galup extrae el parámetro $N_{c i}$ de la curva de la varianza de la corriente en sub-umbral, luego este valor se usa junto con la ecuación (8) para calcular el parámetro de las medidas hechas en inversión fuerte, tanto para saturación como para tríodo. Pero el perfil de dopaje, que determina $N_{c i}$ es altamente dependiente de la polarización, tanto de puerta como de drenador-fuente (Yang, 2003). Lo anterior indica que el procedimiento usado por Galup-Montoro para el cálculo de $B_{I_{S O}}$ es incorrecto. Las medidas ratifican las suposiciones hechas acerca del carácter físico de $N_{c i}$ donde éste exhibe un comportamiento impredecible. Además, se usan medidas de transistores con efecto cuerpo, las cuales también modifican el perfil de dopaje. Cabe destacar el método de medición llevado a cabo, el cual usa una estructura de

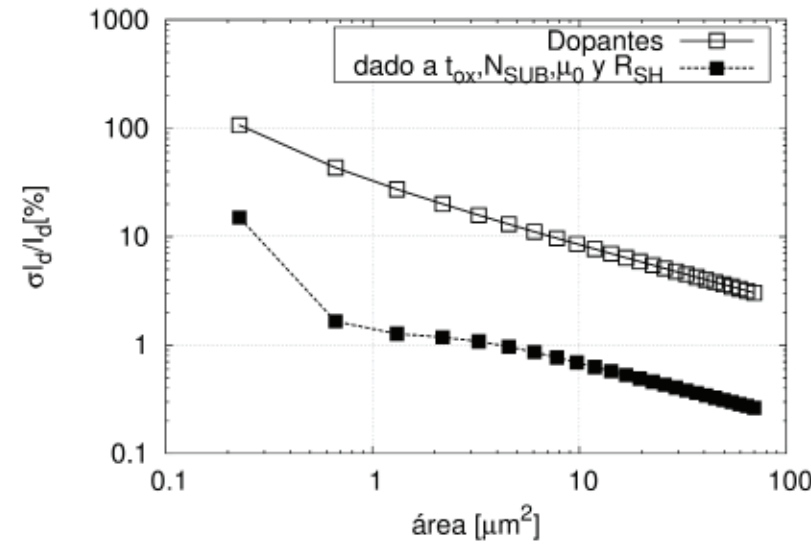

espejo de corriente. Este proceso incluye la toma de medidas por medio de diferentes unidades de prueba, para luego promediarlas y reducir el error. Ni Drennan ni Galup-Montoro tienen en cuenta la medición de las variaciones globales.

\section{Precisión}

La cualidad de precisión de un modelo depende altamente del proceso de fabricación bajo el cual éste fue caracterizado. Por lo tanto, intentar comparar modelos medidos bajo distintas condiciones es algo contraproducente. Para analizar la precisión del modelo Pelgrom en escalas de integración actuales, se comparan las expresiones (7) y (12), que describen la varianza de la corriente en sub-umbral del modelo de Pelgrom y Galup, respectivamente. Ambas tienen dos cosas en común: incluyen básicamente el efecto de dopantes locales en el mismatch que influyen $\sigma_{V_{t}}$ y poseen la misma estructura matemática, ya que $g_{m} / I_{D}$ en sub-umbral es un término constate igual a $1 /{ }_{n} U_{T}$. Sentencia que puede ser confirmada a partir de la figura $3 a$, donde se comparan en simulación los tres modelos discutidos. En este sentido, la precisión de ambos modelos es la misma para el dominio de polarización sub-umbral. Los resultados mostrados en (Galup, 2005) muestran una adecuada aproximación en la estimación del mismatch, por medio del modelo de Galup, para transistores polarizados en inversión débil y de tamaños medianos. Estas mismas tasas de precisión no se obtienen para transistores con dimensiones pequeñas y en aquellos cuyo cuerpo está polarizado, presentándose así valores impredecibles para el parámetro $N_{c i}$.

Realmente, las características eléctricas de los dispositivos de canal corto son muy dependientes de los efectos de borde en el perfil de dopaje. Además, para

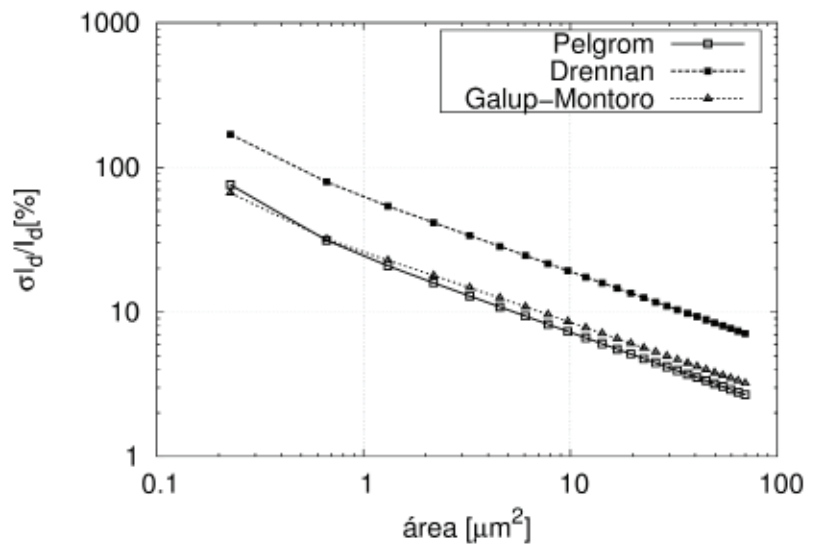

Figura 3. a) Comparación de los tres modelos; b) Efecto de $N_{c i} y$ de $B_{I_{s Q}}$ en el mismatch 
dimensiones nanométricas, las regiones de dopaje del drenador y la fuente están muy cercanas, tornando altamente sensitiva la capa de deflexión debajo del canal. Para analizar la pertinencia de la inclusión de $B_{I_{\mathrm{so}}}$, se simula el efecto que tienen las variaciones despreciadas en la ecuación (5), que para este caso corresponden a $t_{o x}$, $N_{S U B}, \mu_{O}$, y $r_{s h}$ basadas en los modelos worst-case otorgados por el fabricante del CI. En la figura $3 b$ se observa una tendencia altamente lineal para áreas algo mayores a $1 \mu \mathrm{m}^{2}$. Por lo tanto, es posible que la inclusión de $B_{I_{S Q}}$ modele acertadamente estos efectos para dispositivos medianos y grandes.

El modelo Drennan al haber sido ya usado en la industria como el modelo Pelgrom, indicaría lo adecuado, y por ende lo preciso que resultaría ser en la práctica. Pero la evaluación del modelo de Drennan involucra inmediatamente dos factores analizados antes: el modelo de simulación usado y el conjunto de pruebas de caracterización llevado a cabo. Por lo tanto, la precisión del modelo de Drennan es altamente dependiente del contexto bajo el cual se haya derivado.

\section{Utilidad}

La sencillez y adecuada precisión de su tiempo convirtieron a Pelgrom en un modelo estándar en la industria e indudablemente en el modelo más útil, hasta el momento, de la microelectrónica. La utilidad del modelo Pelgrom se fundamenta en una premisa esencial: el aumento de área del dispositivo disminuye proporcionalmente su variabilidad. A pesar de que esta consigna se haya usado ampliamente en el diseño, no resuelve preguntas que buscan controlar más a nivel físico y de circuito el fenómeno del mismatch. Además, el diseño ha migrado a distintas regiones de polarización y los procesos de fabricación han incluido nuevas estrategias de implantes de impurezas que no pueden ser modelados por las mismas ecuaciones que derivaron este modelo. Otro aporte esencial del modelo Pelgrom consiste en la inclusión de las variaciones globales en los procesos que involucran el diseño del layout, y que fundamentaron técnicas como el centroide común. La utilidad del modelo Pelgrom es indudable: las varianzas de los parámetros en relación con el dimensionamiento del dispositivo pueden ser utilizadas para estimar y diezmar el efecto del mismatch como también para proponer nuevos modelos.

La utilidad del modelo Drennan se enfoca en la etapa de verificación del diseño, pero no en el diseño mismo, pues las expresiones en (3) sólo pueden evaluarse por medio de simulación, partiendo de un dimensionamiento previo del circuito. Para efectuar estimaciones manuales de mismatch, se debe recurrir a modelos como el de Pelgrom. Además, bajo el modelo de Drennan no pueden efectuarse fácilmente compromisos como mínima área y variabilidad, dado a que en cada parámetro está ligada una sensibilidad dependiente del dimensionamiento. La evaluación de este tipo de compromisos implicaría la caracterización de la sensibilidad para un amplio rango de dimensiones, en conjunto con técnicas computacionales. El modelo de Drennan no propone una estrategia distinta que pueda llevarse a nivel de layout, por lo que el circuito es transparente a los efectos de la disposición de los elementos en el layout.

El modelo de Galup-Montoro es útil en la medida en que el modelo $A C M$ se asocie con las herramientas de simulación y diseño. El modelo $A C M$ ya se ha implementado en simulación (Filho, 1999) y sus parámetros se han extraído del modelo BSIM3v2 por dos métodos: confrontación de ecuaciones y simulación (Coitinho, 2001). A pesar de eso, no existen herramientas comerciales que tengan a su disposición la implementación del $A C M$, por lo que contrastar resultados en el proceso de diseño bajo dos modelos diferentes se convierte en un proceso tedioso. La principal utilidad de Galup es la obtención de un modelo físico, continuo y relativamente sencillo que describe acertadamente el fenómeno del mismatch. Por último, es bueno establecer que en este modelo no se consideran las variaciones globales, por lo tanto, resulta poco útil para simulaciones posteriores del layout.

\section{Adaptabilidad}

En el modelo Pelgrom, se ha presentado una disminución en el valor de $A_{V_{T}}$ con la reducción del tamaño de los transistores MOS $^{T}$ (Kinget, 2005). Por lo tanto, un dispositivo que ocupe un área constante mejorará su precisión en futuros procesos sub-micrón. Pero esta tendencia de mejoramiento se ve contrastada con el aumento de la densidad de integración, ligada a una menor área por dispositivo, lo que conduce a un aumento en la variabilidad. La disminución del tamaño característico es cuadrática comparada con la disminución lineal de $A_{V_{T}}$ (Kinget, 2005). Además, las nuevas tendencias en circuitos promueven un bajo $\left(V_{G S}-V_{t}\right)$, por lo que una proporción alta de $\left(g_{m} / I_{D}\right)$, tal que los errores en $V_{t}$ debido a los fenómenos inherentes al canal comienzan a ser considerables. En consecuencia, la variabilidad de muchos parámetros se torna más dependiente a la longitud de canal que del ancho del transistor, razón por la cual el exponente de $1 / L$ debe ser aumentado con respecto al exponente de $1 / W$. 
La insuficiencia del modelado en Pelgrom es notoria en tecnologías de escalas sub-micrón. Para estimar el mismatch en la corriente de procesos futuros, se debe llevar a cabo una nueva derivación del modelo basada en ecuaciones de canal corto. A pesar de esto, la esencia de la ecuación (1) se seguirá manteniendo para muchos de los parámetros durante los años futuros. En general, se puede decir que la escalabilidad en el modelo Pelgrom es limitada.

En Drennan se siguen manteniendo la dependencia del dimensionamiento con la varianza de los parámetros dispuesta por Pelgrom, pero se le agrega un nuevo componente: las sensibilidades. El análisis entre las sensibilidades y el escalamiento muestra un aumento de éstas conforme se reduce el dimensionamiento. Esta deducción es consecuente con el recurrente concepto del mismatch, por lo que el escalamiento produce resultados coherentes en el modelo Drennan. Se podría afirmar de esta manera que el modelo de Drennan es altamente escalable.

Finalmente se analiza la escalabilidad del modelo de Galup-Montoro. Inicialmente, la ecuación (7) evidencia una tendencia similar a la establecida por Pelgrom, esto puede corroborse a partir de los resultados comparativos de los tres modelos presentados en la figura 3a. El efecto de escalamiento sobre las corrientes $i_{f}$ e $i_{r}$ es mínimo, dado a que éstas son parámetros normalizados. La gran diferencia se establece en $N_{c i}$ el cual es altamente sensible al escalamiento como confirmó el análisis de medibilidad de este modelo. Para adecuarlo a futuras generaciones de proceso, el modelo se torna fácilmente escalable si se incluye la dependencia de los voltajes dreno-fuente $V_{D S}$ y fuente-substrato $V_{S B}$ sobre el parámetro $N_{c i}$. Además si se reconfirma la pertinencia de la inclusión del factor $B_{I_{s o}}$ para modelar los efectos de la variabilidad de otros parámetros como $t_{\text {ох }}, N_{\text {Sив }}$ у $\mu_{O}$.

\section{Conclusiones}

El modelo Pelgrom, al establecer de manera sencilla una tendencia y no un estimativo de variabilidad en el circuito, resulta adecuado como punto inicial para su dimensionamiento. De esta manera, la ecuación (1) fácilmente acotaría el espacio de diseño en el contexto de la determinación de las mínimas áreas permisibles, de forma tal que etapas posteriores de dimensionamiento sean garantizadas en el marco del diseño para fabricación. Por su parte, los bajos niveles de precisión del modelo Pelgrom en tecnologías sub-micrón, hacen de éste un estimativo inadecuado para las fases que involucran la validación del diseño. La sobreestimación del mis- match en las etapas de validación del diseño origina un re-dimensionamiento que tenderá a exceder el área a niveles poco permisibles. Paralelamente, una sub-estimación del mismatch conduce a resultados de rendimiento sobreestimados que enaltecen la robustez del circuito en conjunto con las herramientas y metodologías asociadas al diseño.

De esta manera, en búsqueda de una adecuada estimación para la validación final del diseño y habiendo descartado el modelo de Pelgrom para tal propósito, el diseñador se encuentra ante los dos modelos restantes estudiados en el presente trabajo. Por un lado, el modelo de mismatch fundamentado en el modelo $A C M$ resulta ser un estimativo relativamente sencillo y considerablemente adecuado para la fase de verificación y re-dimensionamiento del circuito, pero presenta dos limitantes importantes. Primero, no existen las herramientas comerciales que permitan una aplicabilidad global del modelo $A C M$ en el flujo de diseño y entonces se requiere la implementación de por lo menos dos modelos para la fase de diseño. Y segundo, la expresión que propone la varianza de la corriente se reduce a la establecida por Pelgrom en transistores polarizados en sub-umbral, representando una transparencia en la aplicabilidad de estos dos modelos para diseños que requieran bajo consumo de potencia. $\mathrm{Al}$ ser $A C M$ un modelo relativamente reciente, requiere de desarrollos posteriores que lo extiendan para transistores en subumbral y fenómenos no tenidos en cuenta como las variaciones en el óxido de puerta. Por otro lado, Drennan propone un modelo lo suficientemente complejo como para no usarlo en la etapa de diseño, dado que su expresión involucra de manera distinta las sensibilidades de cada uno de los parámetros físicos relevantes. El tedioso cálculo ligado a la determinación de las dimensiones en la expresión (3), hacen de éste un modelo inadecuado para establecer compromisos entre variables de diseño ligadas al dimensionamiento con las especificaciones de rendimiento y desempeño establecidas. Pero la expresión en (3) se torna fácilmente calculable cuando las dimensiones y polarizaciones han sido definidas previamente, es decir, cuando ya se cuenta con un diseño preliminar para verificación.

De esta manera, la selección de los modelos a usar requiere el análisis bajo marcos de diseño distintos. Primero, dadas unas exigencias iniciales de circuito a nivel de variabilidad, el área debe ser restringida a los valores mínimos permisibles. Este proceso al requerir sólo una tendencia de variabilidad y excluyéndose del análisis de un circuito ya dimensionado, puede bordarse ampliamente por medio de un modelo como Pelgrom. Por consiguiente, el diseño final al involucrar altas exi- 
gencias de precisión en la estimación de su desempeño y rendimiento, requiere un modelo bastante acorde con los resultados de silicio. Al haber descartado tanto Pelgrom como $A C M$ para este propósito, convierten a Drennan en el modelo adecuado para la validación del diseño. Debe ser clara la idea de que la elección de un modelo de mismatch está ampliamente ligada al contexto bajo el cual se requiera determinar la variabilidad. Por ejemplo, para transistores polarizados en saturación, la expresión (5) que propone el modelo ACM podría usarse en la etapa de verificación, ya que describe adecuadamente la varianza de la corriente en esta región. De otra manera, la variabilidad de inversión débil al ser más alta que la de saturación, requiere un proceso de estimación más riguroso. Dependiendo de la metodología de diseño adoptada, la elección de los modelos del mismatch debe adecuarse a las intenciones y medios presentes de cada una de las etapas del flujo de diseño.

\section{Referencias}

Coitinho R., Spiller L., Schneider M., Galup-Montoro C. A Simplified Methodology for the Extraction of the ACM MOST Model Parameters, Integrated Circuits and Systems Design, $14^{\text {th }}$ Symposium, pp. 136-141, 2001.

Croon J., Rosmeulen M., Decoutere S., Sansen W., Maes H. An Easy-to-Use Mismatch Model for the MOS Transistor. SolidState Circuits, IEEE Journal, 37(8):1056-1064, agosto de 2002.

Drennan P., McAndrew C. Understanding MOSFET Mismatch for Analog Design. Solid-State Circuits, IEEE Journal, 38(3):450456, marzo de 2003.

Filho O.G. Um Modelo Compacto do Transistor MOS para Simulacão de Circuitos, (Ph.D.) dissertation, UFSC, Florianopolis Brazil, septiembre de 1999.
Galup-Montoro C., Schneider M., Klimach H., Arnaud A. A Compact Model of MOSFET Mismatch for Circuit Design. SolidState Circuits, IEEE Journal, 40(8):1649-1657, agosto de 2005.

Galup-Montoro C., Schneider M., Cunha A., De Sousa F., Klimach H., Siebel O. The Advanced Compact MOSFET (ACM) Model for Circuit. Analysis and Design, Custom Integrated Circuits Conference, 2007. CICC '07. IEEE, pp. 519-526, septiembre de 2007.

Kinget P. Device Mismatch and Tradeoffs in the Design of Analog Circuits, Solid-State Circuits, IEEE Journal, 40(6):1212-1224, junio de 2005.

Lakshmikumar K., Hadaway R., Copeland M. Characterisation and Modeling of Mismatch in MOS Transistors for Precision Analog Design. Solid-State Circuits, IEEE Journal, 21(6):10571066, diciembre de 1986.

Lei J., Lima-Filho P., Styblinski M., Singh C. Propagation of Variance Using a New Approximation in System Design of Integrated Circuits, en: Aerospace and Electronics Conference, 1998, NAECON 1998, Proceedings of the IEEE 1998 National, julio de 1998, pp. 242-246.

Pelgrom M., Duinmaijer A., Welbers A. Matching Properties of MOS Transistors. Solid-State Circuits, IEEE Journal, 24(5):14331439, octubre de 1989.

Serrano-Gotarredona T., Linares-Barranco B., Velarde-Ramirez J. A Precise CMOS Mismatch Model for Analog Design from Weak to Strong Inversion. Circuits and Systems, 2004, ISCAS '04. Proceedings of the 2004 International Symposium, pp. I-7536, vol. 1, mayo de 2004.

Yang H., Macary V., Huber J., Min W.G., Baird B., Zuo J. Current Mismatch Due to Local Dopant Fluctuations in MOSFET Channel. Electron Devices, IEEE Transactions, 50(11).2248-2254, noviembre de 2003.

\section{Semblanza de los autores}

Jorge Johanny Sáenz-Noval. Ingeniero electrónico de la Universidad Industrial de Santander en Bucaramanga, Colombia. Es asesor de investigación del grupo de diseño en circuitos integrados CIDIC, en el cual elabora en conjunto, un receptor inalámbrico en un solo chip. Sus áreas de investigación son: metodologías de diseño para fabricación (DFM), herramientas de diseño electrónico asistido por computador EDA y circuitos de baja potencia. Actualmente realiza sus estudios de maestría en ingeniería electrónica en la Universidad de São Paulo.

Élkim Felipe Roa-Fuentes. Ingeniero eléctrico de la Universidad Industrial de Santander en Bucaramanga, Colombia. Obtuvo el grado de maestro en la Universidad de São Paulo en Brasil con la tesis Una metodología para el diseño de un amplificador de bajo ruido CMOS. Es director del grupo de diseño en circuitos integrados CIDIC. Fue profesor asistente de la Universidad Industrial de Santander en los cursos de diseño de circuitos analógicos, microelectrónica I y microelectrónica II. Actualmente está realizando sus estudios de doctorado en Purdue University, EU. 